\title{
GAS AND WATER CRESTING TOWARDS HORIZONTAL WELLS
}

\author{
J. F. McCARTHY'
}

(Received 9 July 1991; revised 24 March 1992)

\begin{abstract}
The hodograph method for flow in porous media is used to study the problem of simultaneous gas and water cresting towards a horizontal well in a thin oil column reservoir. Shapes of the free interfaces are found and an expression for the optimal placement of the well with respect to the interfaces is given. In addition, a numerical technique is used to find the shape of the free interface and values of critical heights for the case of water cresting towards a horizontal well beneath an impermeable plane.
\end{abstract}

\section{Introduction}

The Gippsland Basin, off the coast of Victoria, contains several examples of gas-condensate reservoirs underlaid by thin oil accumulations. For example, the Snapper field originally contained a column of oil of thickness varying between 4 and 8 metres, extending over most of the field and containing a substantial volume of oil. Also, the recently-developed Bream field is producing from a 13 metre oil column. These so-called oil "pancakes" are bounded below by aquifers with a strong bottom water drive, and above by large gas caps. In such a situation, conventional production from a vertical well perforated in the oil zone is plagued by coning problems (see for example $[3,6]$ ), with both water and gas breaking into the well, leading to loss of oil production and loss of oil to residual entrapment.

Coning towards vertical wells is caused by potential gradients being set up

${ }^{1}$ BHP Research - Melboume Laboratories, P.O. Box 264, Clayton, Vic. 3168, Australia.

(C) Australian Mathematical Society, 1993, Serial-fee code 0334-2700/93 
in the flowing oil layer which draw gas and water towards the well. There is a critical rate of oil production above which unwanted fluids break through into the well. Below the critical rate, a stable cone of stationary fluid exists whose height is determined by the equilibrium between the potential gradients due to the oil flow and the gravity forces due to the density difference between the fluids. The critical rate is the flowrate corresponding to the highest stable cone.

It has been suggested that the use of horizontal wells may act to reduce the adverse effects of coning [7]. An illustration of a horizontal well is shown in Figure 1(a). With modern drilling techniques, horizontal wells of up to 1500 metres in length can be drilled with precision. Figure $1(\mathrm{~b})$ is a cross-sectional schematic showing the mathematical idealisation of the physical reservoir which is used for the calculations described in this paper. The horizontal well is represented by an infinite line sink, thus neglecting end effects.

Compared to a vertical well, a horizontal well offers a greater surface area for production in the oil zone, leading to improved productivity. Also, improvements in the critical rate of up to a factor of 7 have been reported for horizontal wells in thin oil zones [7]. Intuitively, this improvement is caused by the potential gradients being less severe in the vicinity of a horizontal well than a vertical well. The gas and water are drawn into a crest shape (see Figure 1(b)), rather than a cone, and the phenomenon is called "cresting".

The problem of calculating critical rates is complicated by the interdependence of the oil-zone potential distribution and the shape of the fluid interfaces. It is essentially a free-boundary problem. The earliest work on critical rates was done by Muskat and Wyckoff [11]. They considered the problem of water coning towards a vertical well, with the well being represented by a point sink at the top of the oil zone. Their main approximation was to calculate the potential function in the oil zone assuming horizontal radial flow and ignoring the presence of the cone. Because of this approximation, their calculated critical rates are between 20 to 25 percent too high.

In another approach, Dupuit's well-discharge formula [6] can be used to derive a relation for the critical rate which takes into account the presence of the cone. This approach was used, e.g., by Meyer and Garder [10]. In their analysis, the critical situation is assumed to occur when the cone reaches the bottom of the well. As a consequence, the derived relations depend on near-well effects, in particular, on the well radius. In fact it is known that the apex of the last stable cone remains at a distance from the bottom of the well which is much greater than the well radius. Thus, the validity of these relations is dubious. They give critical rates which are too low by about a factor of two. 


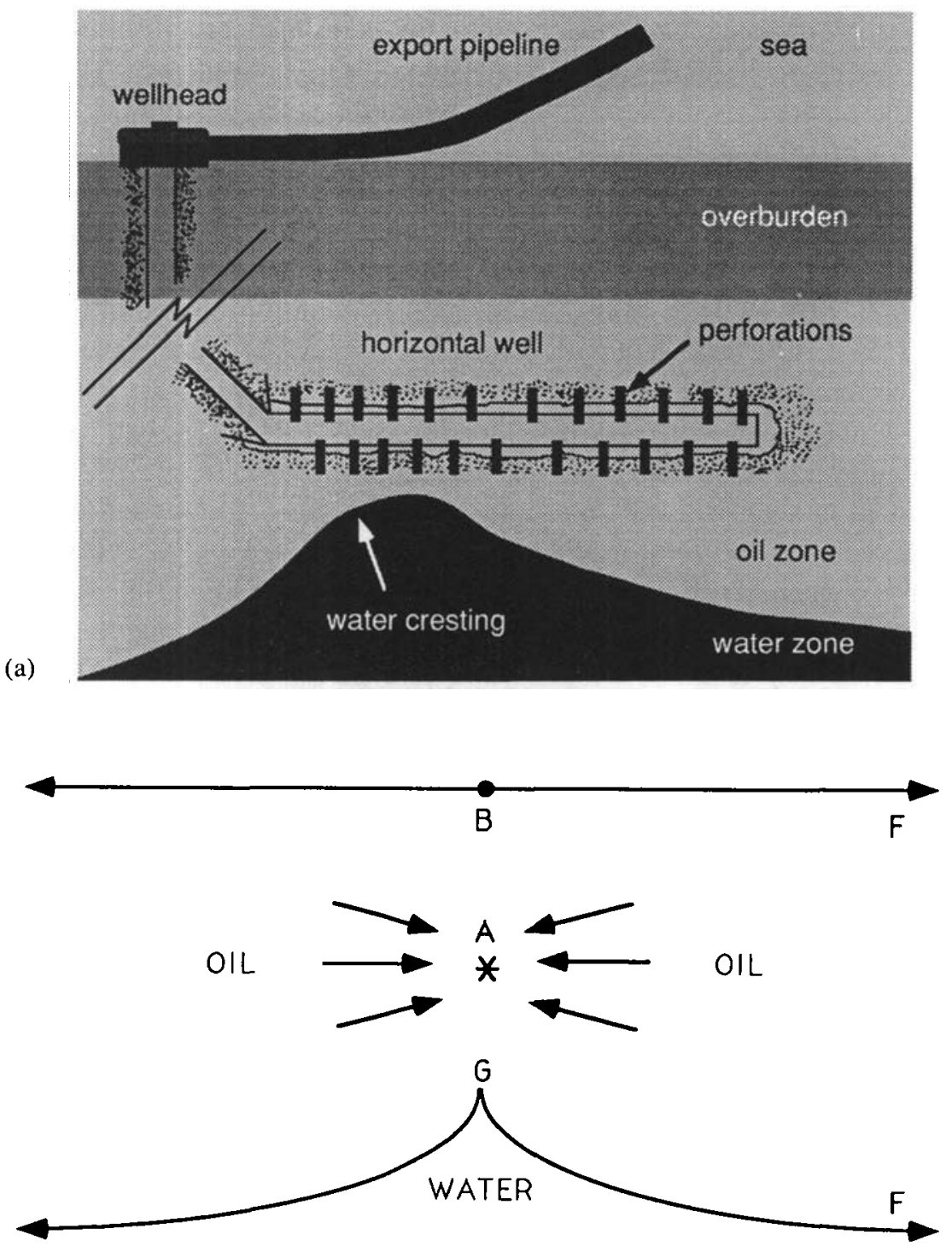

(b)

FIGURE 1. (a) Diagram illustrating water cresting towards a horizontal well beneath an impermeable overburden. (b) Cross-sectional schematic of water cresting towards a horizontal well beneath an impermeable plane. 
A few papers exist which treat the full mathematical free-boundary problem, taking into account the effect of the cone shape on the fluid potential function. Blake, Lucas and Kucera [4] used a boundary-integral formulation to calculate numerical solutions. Their results show a cusping of the cone as the critical rate is approached. In two dimensions, the hodograph method (see e.g. [1]) can be used to find analytical solutions in some special cases. The two-dimensional nature of the solutions means that they are most appropriately applied to the problem of cresting towards an infinitely-long horizontal well. Bear and Dagan [2] used a hodograph method to find the shape of the interface and the height of the crest for the cases of a sink in an unconfined oil zone and a sink on a horizontal impermeable plane. (Note: Actually, they were interested in groundwater flow, and considered the case of salt water being drawn into a fresh water sink, but the two problems are equivalent. There are many related problems in groundwater flow which have been solved analytically by Polubarinova-Kochina [12] using the hodograph method.) In these special cases, Bear and Dagan were able to obtain analytical solutions for the full non-critical problem in which there is a stagnation point below the sink and a point of inflection somewhere on the free- interface. Their solutions show that as the critical case is approached the shape of the free interface tends to a cusp under the sink. These solutions were used by Giger [8] in his paper on analytic two-dimensional models of water cresting before breakthrough for horizontal wells. He also considered some other cases in which analytical solutions can be found; specifically, cases in which the reservoir has impermeable boundaries on three sides and production is via a piston-like mechanism.

In this paper, the hodograph technique is applied to the situations depicted in Figures 1(b) and 2, i.e. a sink below a horizontal impermeable plane, and a sink between two free interfaces. As indicated in the figures, only the critical case is considered, with the free interfaces forming a cusp in line with the sink. This is the case of most practical importance. For Figure 2, it corresponds to the problem of locating the optimal position of the sink with respect to the critical rate for gas and water cresting. This problem is addressed in Section 5. The hodograph method is used to find analytical expressions for the shapes of the two free interfaces, and the heights $\overline{A G}$ and $\overline{A H}$ are calculated.

The situation depicted in Figure 1(b) incorporates the special cases considered by Bear and Dagan, i.e. $\overline{A B}=0$ corresponds to a sink on a horizontal plane and $\overline{A B} \rightarrow \infty$ corresponds to a sink in an unconfined oil zone. We review these solutions in Section 3, restricting ourselves to the critical case for consistency. For the case when $\overline{A B}$ is finite, the problem cannot be treated analytically 


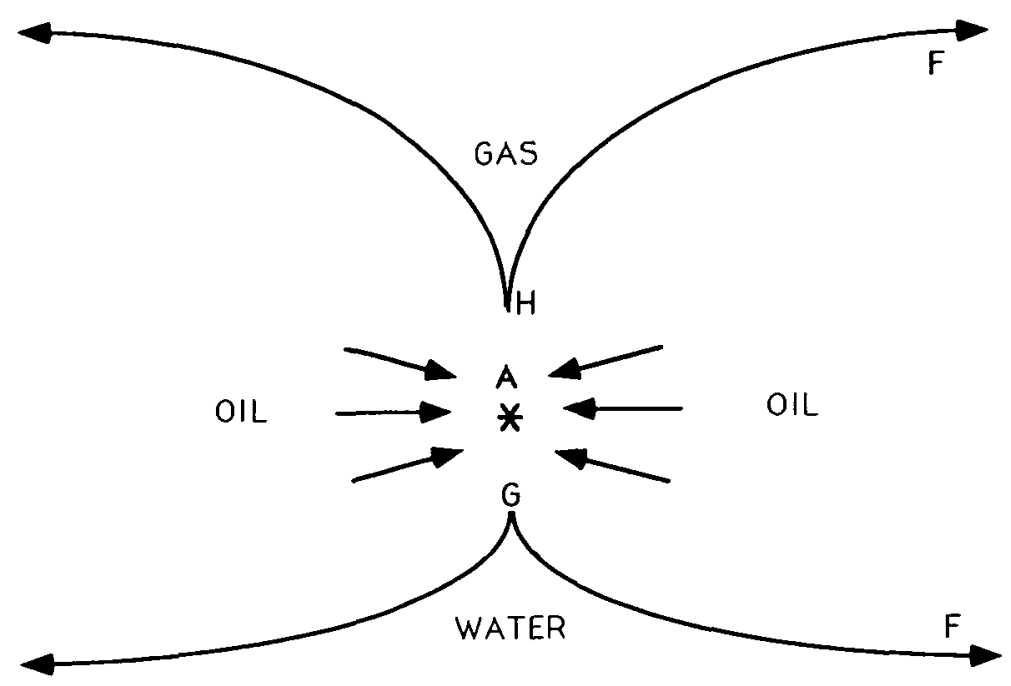

FIGURE 2. Cross-sectional schematic of simultaneous gas and water cresting towards a horizontal well.

because of the right-angled comer singularity in the fluid flow at point $\mathrm{B}$. It is necessary to use a numerical technique, as is done in Section 4. Cusp heights, sink heights and interface shapes are calculated for various positions of the sink relative to the horizontal plane.

The numerical technique used in Section 4 is the same as that introduced in fluid dynamics for the solution of free-surface flows due to a submerged source or sink [13]. The fluid dynamics problem is mathematically equivalent to the water cresting problem considered in this paper, except that the freesurface condition is given by Bernoulli's equation rather than the more simple condition which can be derived for flow in porous media (see Section 2). This necessitates the use of numerical methods. A succession of papers has appeared on the fluid dynamics problem (e.g., [5, 9, 13, 14]), considering such cases as stagnation-point flows, cusped free-surface flows, a sink on an inclined (rather than a horizontal) impermeable plane, and the zero-gravity case. Many of these cases have interesting analogies in the water cresting problem; e.g., for cusped free surface flows, solutions are observed at a unique Froude number, analogous to the critical rate for cresting. 
The arrangement of this paper is as follows. In Section 2, the hodograph method for flow in porous media is briefly described. In Section 3, two special cases of cresting towards a horizontal well are reviewed. In Section 4, a numerical technique is used to calculate free interfaces and critical heights for cresting towards a horizontal well beneath an impermeable plane. In Section 5, the problem of simultaneous gas and water cresting towards a horizontal well is treated. Section 6 contains the conclusion.

\section{The hodograph method for flow in porous media}

Darcy's equation for flow in porous media is

$$
q=-\nabla \phi
$$

where $q$ is the specific discharge vector and $\phi$, the velocity potential function, is given by

$$
\phi=\kappa(y+p / \gamma) .
$$

Here $\kappa$ is the hydraulic conductivity, $\gamma$ is the specific gravity of the fluid, $p$ is the pressure at a point, and $y$ is the elevation above some arbitrary level. The horizontal and vertical components, $u$ and $v$, respectively, of the specific discharge vector are given by

$$
\begin{gathered}
u=-\partial \phi / \partial x, \\
v=-\partial \phi / \partial y .
\end{gathered}
$$

Assuming incompressible flow leads to Laplace's equation for the velocity potential,

$$
\nabla^{2} \phi=0 .
$$

By introducing the conjugate harmonic function $\psi$ of $\phi$, called the stream function, a complex potential function, $f$, is obtained, namely

$$
f=\phi+i \psi \text {. }
$$

Let the coordinate plane (illustrated in Figures $1(\mathrm{~b})$ and 2) be called the $z$ plane, where $z=x+i y$. Then the fundamental relation of the hodograph method is,

$$
\partial f / \partial z=-\omega
$$


where

$$
\omega=u-i v
$$

The $\omega$-plane is called the hodograph plane.

In the hodograph plane, the interface between two immiscible liquids of different densities (e.g. oil and water) is given by

$$
u^{2}+(v-K / 2)^{2}=(K / 2)^{2}
$$

where

$$
K=\kappa\left(\gamma_{w}-\gamma_{o}\right) / \gamma_{o}
$$

and $\gamma_{o}$ and $\gamma_{w}$ are the specific gravities of the flowing and stationary fluid phases, respectively (see e.g. [1]). Hence the shape of the interface is given by an arc of a circle in the hodograph plane. Once the conformal transformations mapping the $f$ and $\omega$-planes to the $z$-plane are found, (7) can be integrated to give the shape of the interface in the coordinate plane. This is the essence of the hodograph method. For the related fluid dynamics problem, discussed in the introduction, the free-surface condition is given by Bernoulli's equation and does not map to a simple curve in the hodograph plane (except in the case of infinite Froude number [5], when gravity is neglected). This necessitates the use of numerical methods to solve the problem.

Step by step, the hodograph method for flow in porous media is as follows [2]:

(i) the flow region in the coordinate $z$-plane is mapped onto the complex potential $f$-plane by mapping its boundaries;

(ii) the $z$-plane is mapped onto the hodograph $\omega$-plane;

(iii) the $\omega$-plane is mapped onto the (so called) $\bar{\omega}$-plane by using an inversion transformation

$$
\bar{\omega}=K / \omega
$$

(iv) the flow region in both the $\bar{\omega}$ and $f$-planes is mapped onto the upper half of an auxiliary $\zeta$-plane;

(v) with $\zeta$ as an independent parameter, and using (7), we get

$$
\frac{d z}{d \zeta}=-\frac{\bar{\omega}(\zeta)}{K} \frac{d f}{d \zeta}
$$

This is integrated to find the lengths and shapes of boundary segments in the $z$-plane. 


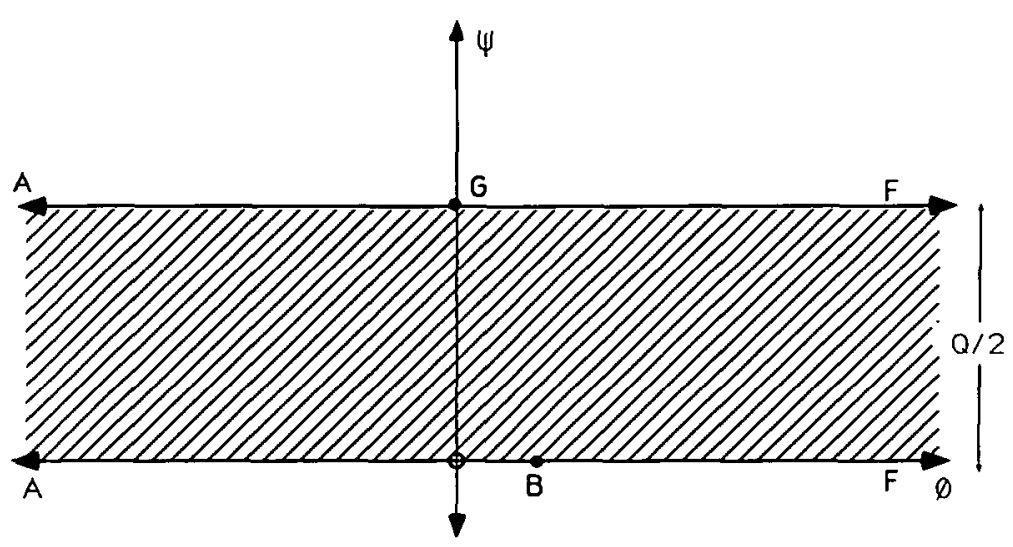

FIGURE 3. Mapping of the flow region onto the complex potential $f$-plane.

The hodograph method is illustrated in Section 3 by reviewing the solution of the water cresting problem in the two cases in which analytical solutions can be found, that is, the case of a sink in an unconfined oil zone, and that of a sink on a horizontal impermeable plane. These solutions provide a framework for the rest of the paper. In particular, they are the limiting cases of the problem considered in Section 4 and provide bounds for the numerical solutions calculated in that section.

\section{Analytical solutions for water cresting}

3.1. Unconfined oil zone. Consider the physical situation illustrated in Figure 1(b): a sink $A$ of strength $Q$ is bounded above by a horizontal impermeable plane $\overline{B F}$, and below by an aquifer $\overline{G F}$. Because of the symmetry of the problem, it is only necessary to consider the right half of the $z$-plane, i.e. the region bounded by $\overline{F B A G F}$ in Figure 1(b). The result of mapping this region to the complex potential plane is shown in Figure 3. $\overline{F G A}$ forms a streamline of the flow, as does $\overline{F \overline{B A}}$. The total flowrate between these streamlines is $Q / 2$.

Consider the case when $\overline{A B} \rightarrow \infty$, i.e. an unconfined oil zone. In this case, the $f$-plane is given by Figure 3 with $B \rightarrow F \rightarrow \infty$. The hodograph plane is shown in Figure 4(a). The free interface $\overline{G F}$ maps to a semicircle of diameter $K$, as given by (9). There is a singularity at the sink point $A$, where the velocity of the flow becomes infinite. Point $A$ maps to the origin in the corresponding 
$\bar{\omega}$-plane, shown in Figure 4(b).

Figure 4(c) shows the auxiliary $\zeta$-plane which was used in the solution of this problem. Using the Schwarz-Christoffel transformation, the $f$-plane is mapped onto the $\zeta$-plane via

$$
f=-\frac{Q}{2 \pi} \log \zeta
$$

The $\bar{\omega}$-plane is mapped onto the $\zeta$-plane via

$$
\bar{\omega}=i\left[1-\left(\frac{1+\zeta}{\zeta}\right)^{1 / 2}\right] .
$$

Using (12), the expression relating the $z$-plane to the $\zeta$-plane is derived to be

$$
\frac{d z}{d \zeta}=\frac{i Q}{2 \pi K} \frac{1}{\zeta}\left[1-\left(\frac{1+\zeta}{\zeta}\right)^{1 / 2}\right]
$$

The shape of the interface $\overline{G F}$ is obtained by integrating (15) for $-1<\zeta<0$ on the real axis. If we make the change of variables $\zeta=-v^{2} /\left(1+v^{2}\right)$ for $0 \leq v \leq \infty$, then the parametric equations of the interface will take the form

$$
\begin{aligned}
& \frac{x K}{Q}=\frac{1}{\pi}\left(\arctan \nu+\frac{1}{v}-\frac{\pi}{2}\right), \\
& \frac{y K}{Q}=-\frac{1}{2 \pi} \log \frac{v^{2}}{1+v^{2}} .
\end{aligned}
$$

The critical height from the top of the cusp to the sink, $\overline{A G}=b_{\text {crit }}$, is obtained by integrating (15) for $-\infty \leq \zeta \leq-1$. It is given by

$$
\frac{b_{c r i t} K}{Q}=\frac{1}{\pi}(1-\log 2)=0.097 .
$$

The calculated interface is shown in Figure 4(d).

3.2. Confined oil zone - sink on a horizontal plane. Consider the physical situation illustrated in Figure 1(b) in the case when $\overline{A B}=0$. This is the case of a sink on a horizontal plane above an oil zone which is bounded below by an aquifer. As before, the appropriate region of the physical $z$-plane is mapped onto the region of the complex potential plane shown in Figure 3, except that in this case $B \rightarrow A \rightarrow-\infty$ because the sink is on the horizontal plane. The mapping onto the hodograph plane is shown in Figure 5(a). This is similar to 
(a)
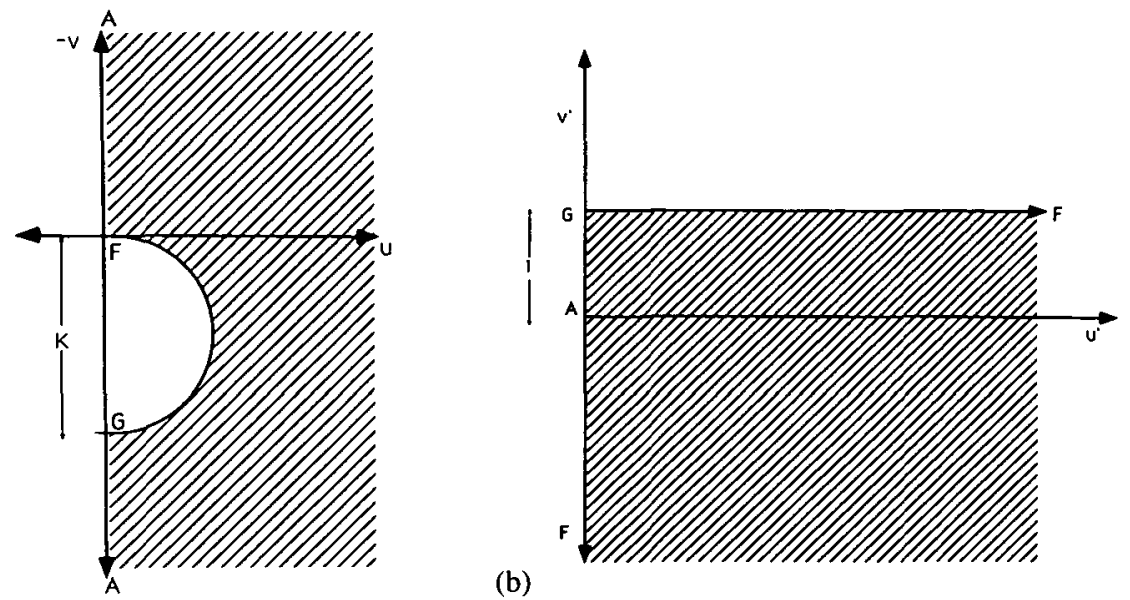

(b)

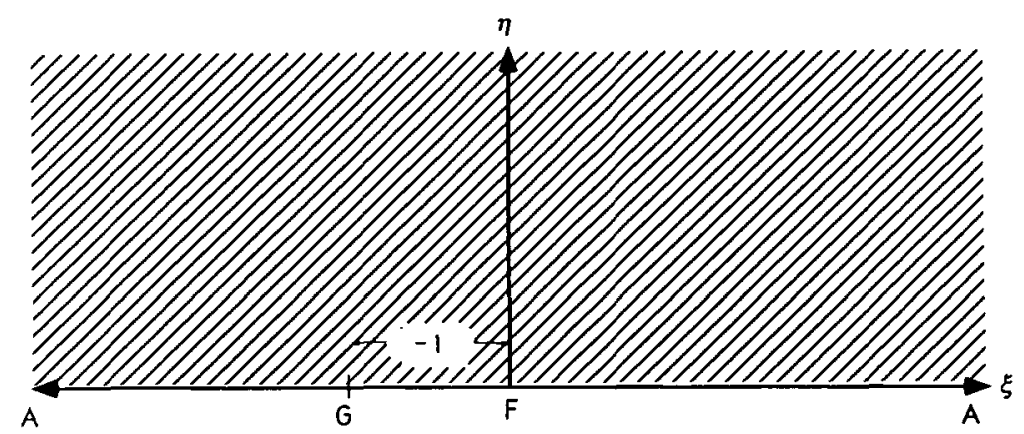

(c) A

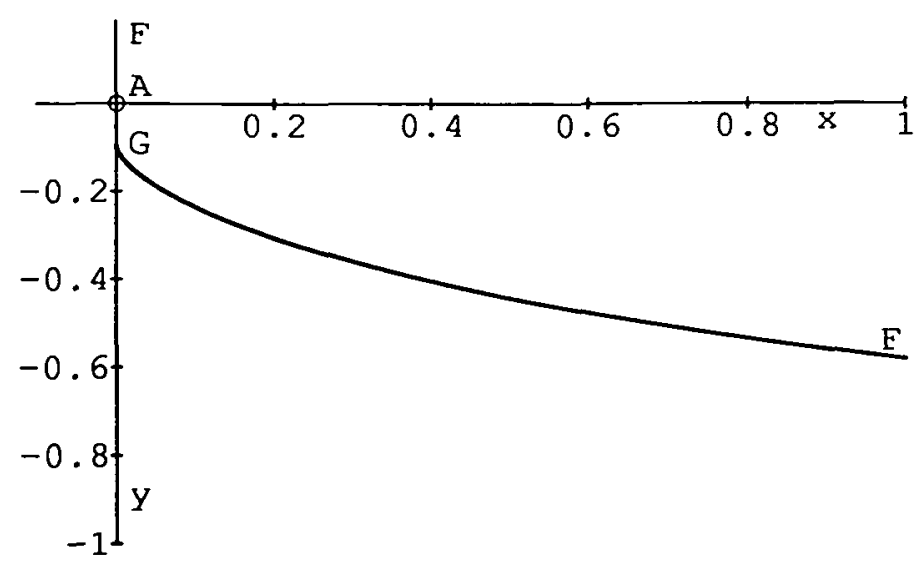

FIGURE 4. The problem of water cresting towards a horizontal well in an unconfined oil zone: (a) the $\omega$-plane, (b) the $\bar{\omega}$-plane, (c) the intermediate $\zeta$-plane, and (d) the calculated free interface in the z-plane. 

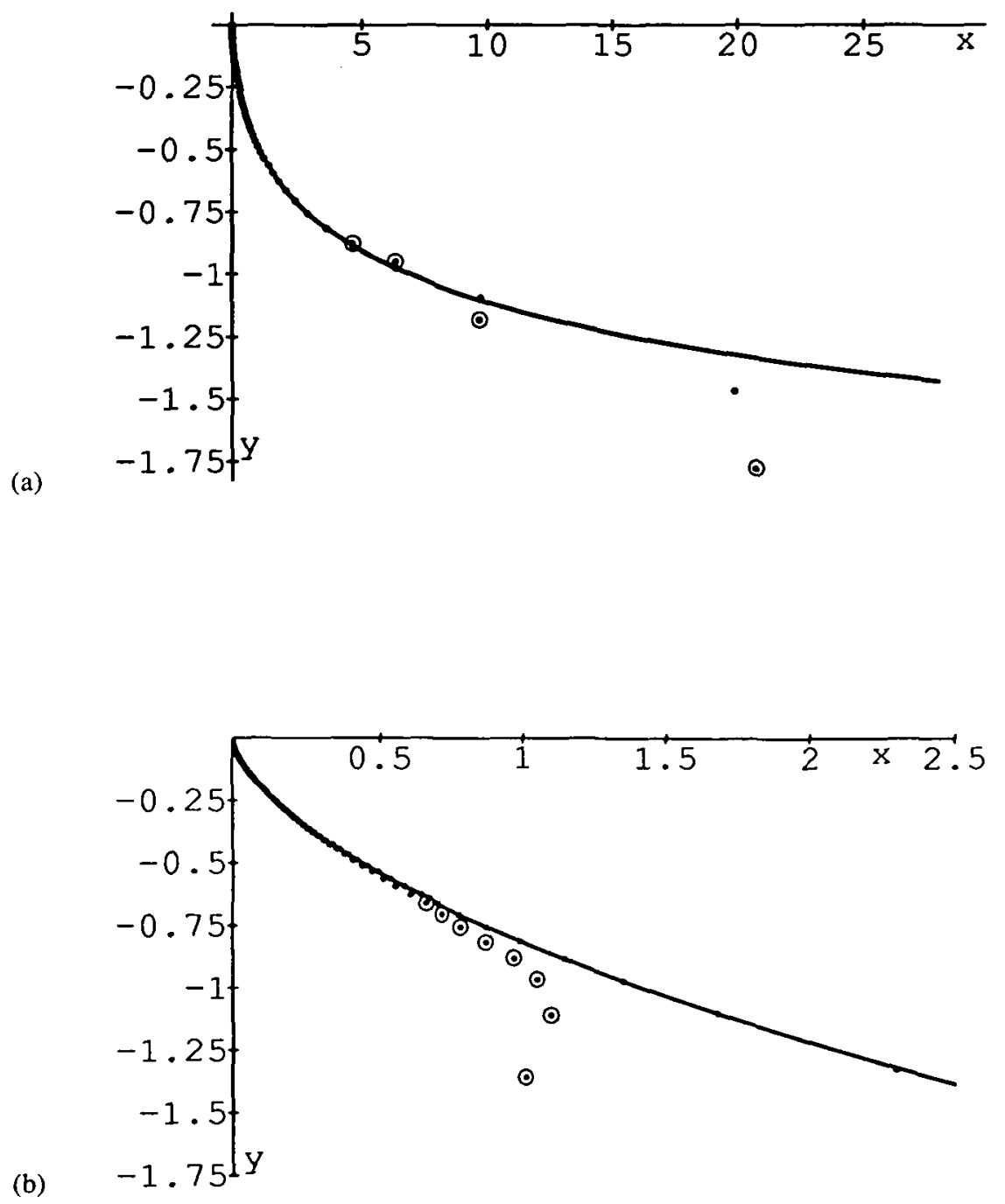

FIGURE 5. The problem of water cresting towards a horizontal well situated on an impermeable plane above the oil zone: (a) the $\omega$-plane, (b) the $\bar{\omega}$-plane, (c) the intermediate $\zeta$-plane, and (d) the calculated free interface in the $z$-plane. 
Figure 4(a), except that now there is no flow in the upper half of the hodograph plane because all the oil flows upwards towards the sink. The corresponding $\bar{\omega}$-plane is shown in Figure 5(b).

The auxiliary $\zeta$-plane which was used in the solution of this problem is shown in Figure 5(c). The $f$-plane is mapped onto the $\zeta$-plane via

$$
f=\frac{Q}{2 \pi} \log \zeta
$$

The $\bar{\omega}$-plane is mapped onto the $\zeta$-plane via

$$
\bar{\omega}=\frac{2}{\pi} \operatorname{arcsinh} \zeta^{1 / 2} .
$$

Using (12), the expression relating the $z$-plane to the $\zeta$-plane is derived to be

$$
\frac{d z}{d \zeta}=-\frac{Q}{\pi^{2} K}\left(\frac{\operatorname{arcsinh} \zeta^{1 / 2}}{\zeta}\right)
$$

The shape of the interface $\overline{G F}$ is found by integrating (21) for $-\infty \leq \zeta \leq-1$. If we let $\zeta=\sinh ^{2}(\nu+i \pi / 2)$ for $0 \leq \nu \leq \infty$, then the parametric equations of the interface will take the form

$$
\begin{aligned}
& \frac{x K}{Q}=\frac{2}{\pi^{2}} \int_{0}^{\nu} \nu \tanh \nu d \nu, \\
& \frac{y K}{Q}=-\frac{1}{\pi} \log \cosh \nu .
\end{aligned}
$$

The critical height from the top of the cusp to the sink is obtained by integrating (21) for $-1 \leq \zeta \leq 0$. It is given by

$$
\frac{b_{c r i t} K}{Q}=\frac{1}{\pi} \log 2=0.2206 .
$$

The calculated interface is shown in Figure 5(d).

\section{Numerical water cresting solutions - sink below horizontal plane}

In Section 3 the hodograph technique was used to find an analytical expression for the shape of the free-interface $\overline{G F}$ (depicted in Figure 1(b)) for the particular cases when $\overline{A B} \rightarrow \infty$ and $\overline{A B}=0$. When $\overline{A B}$ is finite, the problem cannot 


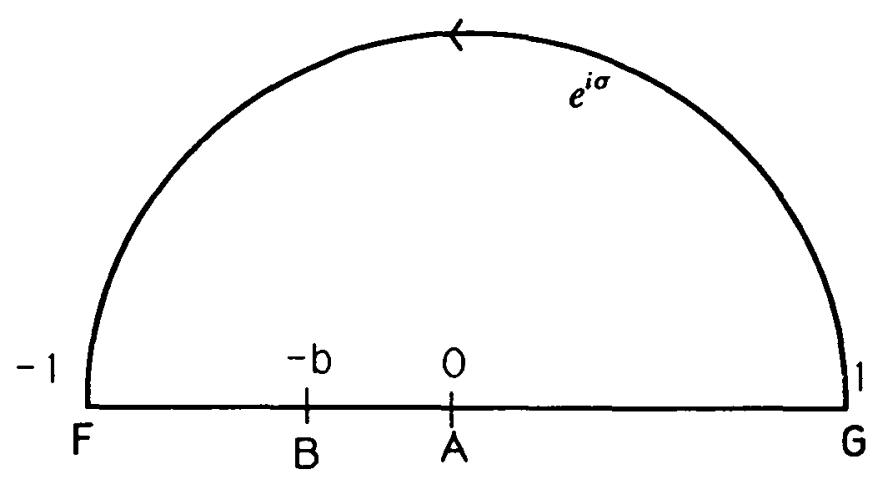

FIGURE 6. The intermediate $t$-plane.

be solved analytically because of the right-angled corner singularity at $B$. It is necessary to use numerical methods, and that is the approach taken in this section.

The numerical technique used here was introduced by Tuck and Vanden Broeck [13] in their solution of the problem of cusp-like free-surface flows due to a submerged source or sink. For their problem, it was not possible to find an analytical solution because of the nature of the boundary condition on the free surface. For our problem, we are fortunate enough to have analytic solutions for the two limiting cases.

To apply the numerical technique, the $f$ and $\omega$-planes are mapped onto a semicircle in the upper-half of the auxilliary $t$-plane, shown in Figure 6. The $f$-plane is mapped via the transformation

$$
f(t)=\frac{Q}{2 \pi} \log \left(\frac{-4 t}{(t+1)^{2}}\right) .
$$

In general, however, there is no such simple transformation for the $\omega$-plane because of the singularities at points $A(t=0)$ and $B(t=-b)$. Instead, the transformation $\omega(t)$ is written in the form

$$
\omega(t)=i K(t+b)^{1 / 2} t^{-1} \sum_{n=1}^{\infty} a_{n} t^{n-1},
$$

where the coefficients $a_{n}$ are to be found. This form has the appropriate singularities, proportional to $t^{-1}$ at $t=0$ and to $(t+b)^{1 / 2}$ at $t=-b$, and is regular 
elsewhere. If all the coefficients $a_{n}$ are constrained to be real, then $\omega(t)$ will be real for $t$ on the real axis between -1 and $-b$, corresponding to the boundary condition that $v=0$ on the horizontal line $\overline{B F}$. Also, $\omega(t)$ will be imaginary for $t$ on the real axis between $-b$ and 1 , corresponding to the boundary conditions that $u=0$ on the segments $\overline{A B}$ and $\overline{A G}$. The remaining boundary conditions, determined by the complex velocity on the free-surface $\overline{G F}$, must be enforced by an appropriate choice of the unknown coefficients $a_{n}$ and the parameter $b$.

If we set $K=1$ for convenience, then the condition for the complex velocity on $\overline{G F}$ is

$$
u^{2}+v^{2}-v=0 \text {. }
$$

A corresponding condition must hold for $\omega(t)$ on the curve $t=e^{i \sigma}, 0<\sigma<\pi$. In addition, the conditions

$$
u=0, \quad v=0 \quad \text { at } \quad \mathrm{F}
$$

and

$$
u=0, \quad v=1 \quad \text { at } \quad \mathrm{G}
$$

are known to hold, and lead to corresponding conditions on $\omega(t)$ at the points $t=-1$ and $t=1$, respectively.

Let us truncate the infinite series in (26) after $N$ terms. This leaves $N+1$ unknowns to be found, i.e. the coefficients $a_{n}$ and the parameter $b$. By introducing $M$ mesh points

$$
\sigma_{l}=\pi \frac{I-1 / 2}{M} \quad(I=1, \ldots, M),
$$

then evaluating the function $\omega$ at these mesh points using (26), and substituting the results into (27), we obtain $M$ non-linear equations for the $N+1$ unknowns. In addition, (28) gives the equation

$$
\sum_{n=1}^{N} a_{n}(-1)^{n-1}=0,
$$

and (29) gives

$$
\sum_{n=1}^{N} a_{n}=(1+b)^{-1 / 2} .
$$

Another equation is obtained by fixing the position of the sink, i.e. by specifying a value for $b$. Hence, there are $M+3$ equations altogether, and $N+1$ unknowns. 
Initially, we set $M=N-2$ and attempted to solve the resulting system of non-linear equations using Newton's method. However, it was not possible to obtain convergence in this way for $b<1$. The reason for this becomes clear if we consider how the numerical method works in the two cases for which we have analytical solutions.

Firstly, let us translate the analytical solutions found in Section 3 from the $\zeta$ variable into the $t$ variable. For problem 3.1 (i.e. $b=1$ ) the equation of transformation from the $\zeta$-plane to the $t$-plane is

$$
\zeta=-(t+1)^{2} /(4 t)
$$

and the equation for $\omega(t)$ becomes

$$
\omega(t)=i(t+1) /(2 t) .
$$

In this case, the coefficients in (26) are the coefficients of the power series expansion about zero of

$$
(t+1)^{1 / 2} / 2
$$

For problem 3.2 (i.e. $b=0$ )

$$
\zeta=-4 t /(t+1)^{2}
$$

and $\omega(t)$ is given by

$$
\omega(t)=i \frac{\pi}{2}\left[\arctan \frac{2 t^{1 / 2}}{t-1}\right]^{-1} .
$$

In this case, the coefficients in (26) are the coefficients of the power series expansion about zero of

$$
\frac{\pi}{2} t^{1 / 2}\left[\arctan \frac{2 t^{1 / 2}}{t-1}\right]^{-1} .
$$

As $t \rightarrow-1$ along the curve $t=e^{i \sigma},(38)$ approaches zero only logarithmically. In fact, it approaches zero as

$$
\left[\log \left(\frac{\cos \sigma+1}{4 \sin (\sigma / 2)+\cos \sigma-3}\right)\right]^{-1} .
$$

Basically, then, it was not possible to obtain convergence of Newton's method with $M=N-2$ for $b<1$ as (31) could not be satisfied due to the slow convergence to zero of the series in (26) as $t \rightarrow-1$. 


\begin{tabular}{|c|c|c|c|c|}
\hline$i$ & \multicolumn{2}{|c|}{$a_{i}$ for case $b=0$} & \multicolumn{2}{c|}{$a_{i}$ for case $b=1$} \\
\hline & Numerical & Exact & Numerical & Exact \\
\hline 1 & 7.8540 E-01 & 7.8540 E-01 & 5.0000 E-01 & 5.0000 E-01 \\
2 & 2.6180 E-01 & 2.6181 E-01 & 2.5000 E-01 & $2.5000 \mathrm{E}-01$ \\
3 & -6.9813 E-02 & $-6.9821 \mathrm{E}-02$ & -6.2501 E-02 & $-6.2500 \mathrm{E}-02$ \\
4 & $3.6569 \mathrm{E}-02$ & $3.6578 \mathrm{E}-02$ & $3.1251 \mathrm{E}-02$ & $3.1250 \mathrm{E}-02$ \\
5 & $-2.3714 \mathrm{E}-02$ & $-2.3725 \mathrm{E}-02$ & $-1.9532 \mathrm{E}-02$ & $-1.9531 \mathrm{E}-02$ \\
10 & $7.4739 \mathrm{E}-03$ & $7.4894 \mathrm{E}-03$ & $5.4547 \mathrm{E}-03$ & $5.4550 \mathrm{E}-03$ \\
20 & $2.7175 \mathrm{E}-03$ & $2.7395 \mathrm{E}-03$ & $1.7296 \mathrm{E}-03$ & $1.7376 \mathrm{E}-03$ \\
30 & $1.5553 \mathrm{E}-03$ & $1.5812 \mathrm{E}-03$ & $8.8457 \mathrm{E}-04$ & $9.1506 \mathrm{E}-04$ \\
40 & $1.0572 \mathrm{E}-03$ & $1.0846 \mathrm{E}-03$ & $4.9463 \mathrm{E}-04$ & $5.8476 \mathrm{E}-04$ \\
50 & $7.8710 \mathrm{E}-04$ & $8.1303 \mathrm{E}-04$ & $3.2806 \mathrm{E}-05$ & $4.1440 \mathrm{E}-04$ \\
\hline
\end{tabular}

TABLE 1. Comparison of numerical $(N=50)$ and exact values for the coefficients of the power series expansion contained in the expression for the complex velocity for the cases $b=0$ and $b=1$.

By setting $M=N-1$ and not imposing (31), convergence was quickly obtained. Most of the calculations described in this section were done with $N=50$.

The coefficients obtained numerically for the cases $b=1$ and $b=0$ are shown in Table 1 compared with the exact values obtained from the series expansion of (35) and (38), respectively. The correspondence is very good in both cases. However, if the series contained in (31) is summed for both sets of coefficients up to $N=50$ then, while the answer is zero for case $b=1$, it is -0.749 for case $b=0$. This illustrates the slow convergence of (38) to zero as $t \rightarrow-1$. As a consequence, the numerical solutions obtained in this section improve in accuracy as $b \rightarrow 1$ for a given value of $N$.

Table 2 shows the convergence with increasing $N$ of the numerically calculated cusp height, $\overline{A G}$, to its exact value for the cases $b=1$ and $b=0$. At $N=50$, the value calculated for the case $b=1$ is correct to 7 decimal places, while the value calculated for $b=0$ is correct to only 3 decimal places. Also included in the table, for comparison, are the cusp heights calculated for the cases $b=0.5$ and $b=0.1$ for which we do not have analytical solutions. At $N=50$ these values have converged to 5 decimal places. 


\begin{tabular}{|c|c|c|c|c|}
\hline$N$ & $b=0$ & $b=0.1$ & $b=0.5$ & $b=1$ \\
\hline 10 & 0.23932 & 0.138316 & 0.103150 & 0.09767649 \\
20 & 0.22516 & 0.138309 & 0.103143 & 0.09767458 \\
30 & 0.22222 & 0.138307 & 0.103142 & 0.09767437 \\
40 & 0.22108 & 0.138306 & 0.103141 & 0.09767432 \\
50 & 0.22051 & 0.138305 & 0.103141 & 0.09767431 \\
$\infty$ & 0.22064 & & & 0.09767428 \\
\hline
\end{tabular}

TABLE 2. Convergence of the cusp height calculated using the numerical method with increasing $N$.

Table 3 contains the cusp heights, $\overline{A G}$, and sink heights, $\overline{A B}$, calculated for various values of the parameter $b$ with $N=50$.

The shape of the interface is obtained by numerically integrating

$$
\frac{d z}{d t}=-\frac{1}{\omega} \frac{d f}{d t}
$$

for $t=e^{i \sigma}, 0 \leq \sigma<\pi$. Figure 7 shows the numerical solutions obtained for various values of the parameter $b$. These solutions were calculated using $N=50$, except for the case $b=0$ (curve (a)), for which $N=100$ was used. Also included in the figure are the exact solutions calculated for cases $b=1$ and $b=0$. Out to the distance shown in Figure 7, the numerical solutions fit the exact solutions very closely. However, as illustrated in Figures 8(a) and (b), the numerical solutions diverge from the exact solutions at sufficiently great distances from the sink. Precisely where this divergence occurs depends on both the value of $N$ used and the value of the parameter $b$. The numerical solutions improve as $N$ increases and as $b \rightarrow 1$.

\section{Simultaneous gas and water cresting}

In this section, the situation illustrated in Figure 2 is considered, in which the oil zone is bounded above by a layer of less dense fluid (e.g. gas), and below by a layer of more dense fluid (e.g. water). This corresponds to a situation of considerable practical interest in which a thin column of oil is bounded above by a gas cap and below by an aquifer, as discussed in the introduction. In Figure 2, 


\begin{tabular}{|r|r|r|}
\hline$b$ & cusp height & sink height \\
\hline 0 & 0.2205 & 0 \\
0.1 & 0.1383 & 0.1555 \\
0.2 & 0.1213 & 0.2696 \\
0.3 & 0.1123 & 0.4105 \\
0.4 & 0.1067 & 0.6011 \\
0.5 & 0.1031 & 0.8761 \\
0.6 & 0.1008 & 1.3036 \\
0.7 & 0.0992 & 2.0433 \\
0.8 & 0.0983 & 3.5785 \\
0.9 & 0.0978 & 8.3561 \\
1 & 0.0977 & $\infty$ \\
\hline
\end{tabular}

TABLE 3. Cusp heights and sink heights calculated using the numerical method with $N=50$.

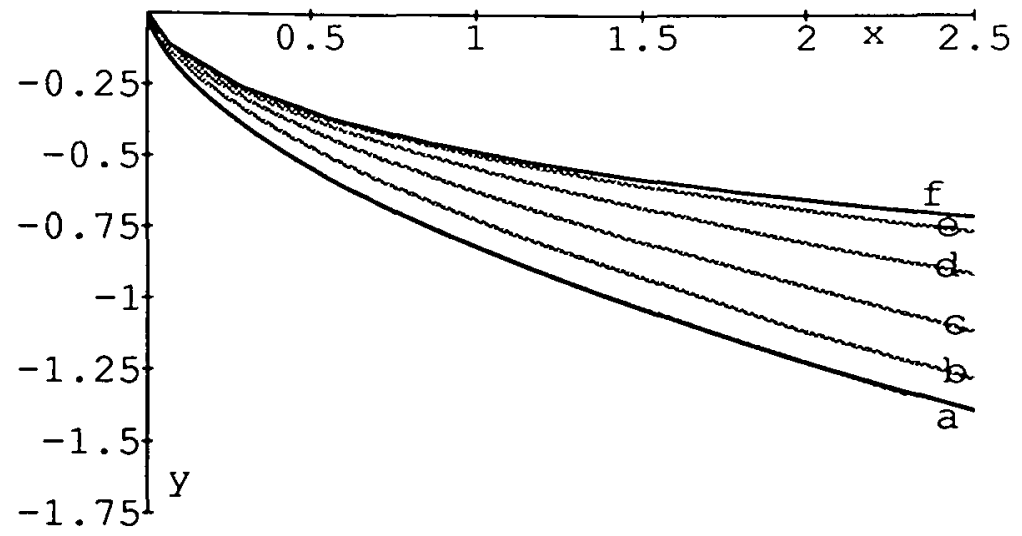

FIGURE 7. Shapes of the free interfaces calculated using the numerical method for the cases of water cresting towards a horizontal well beneath an impermeable plane with (a) $b=0$, (b) $b=0.2$, (c) $b=0.4$, (d) $b=0.6$, (e) $b=0.8$ and (f) $b=1.0$. Also shown (in black) are the exact solutions for cases (a) and (f). 

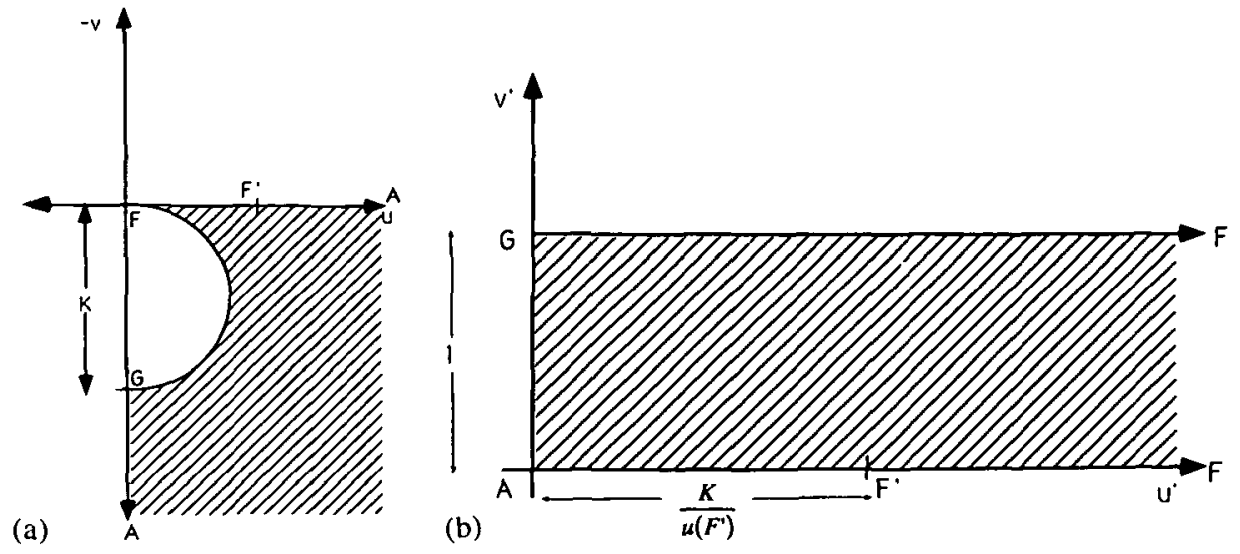

(c)
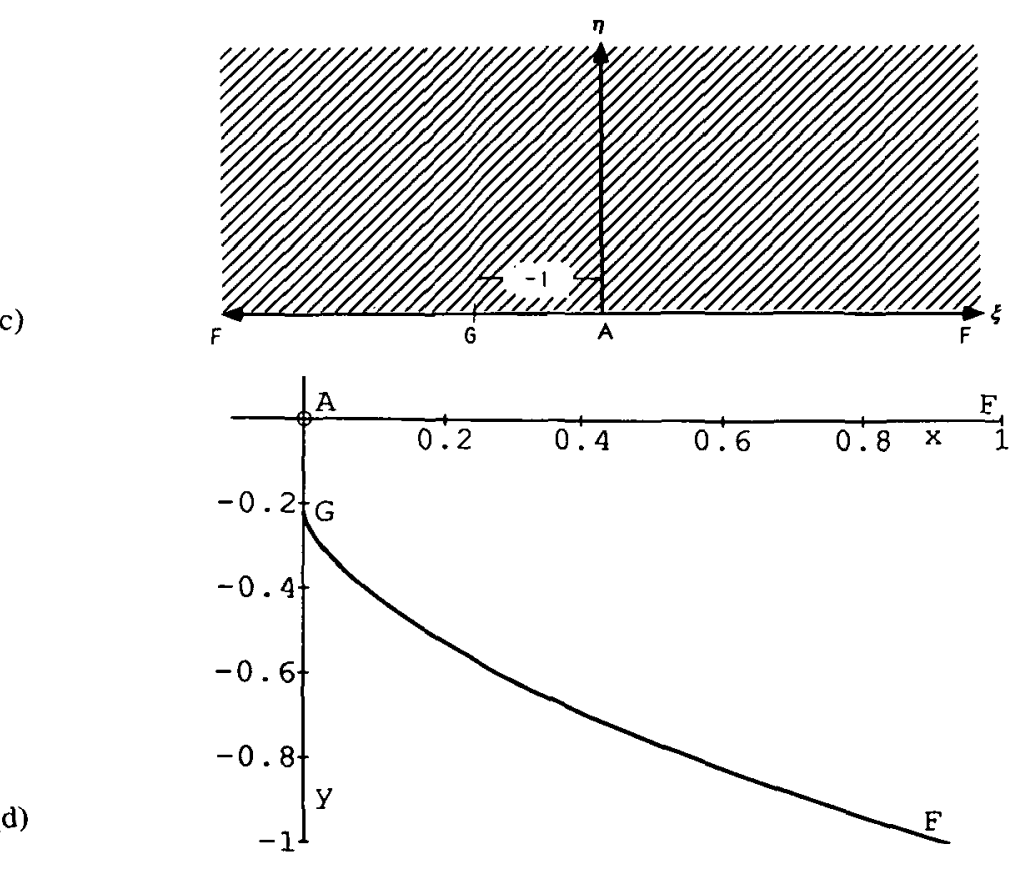

(d)

FIGURE 8. Points on the free interface calculated using the numerical method with $N=50$ (circled dots) and $N=100$ (dots) compared with the exact solution (black line) for the case of water cresting towards a horizontal well beneath an impermeable plane with (a) $b=1$ and (b) $b=0$. 
both the gas-oil interface and the water-oil interface form a cusp in line with the sink, i.e. the situation depicted is critical for both gas and water cresting. The solution of this problem locates the optimum position of the sink with respect to the critical rate for cresting. This is the case which we now examine.

As in Section 3, the $z$-plane is mapped onto the region of the complex potential plane shown in Figure 3, except that in this case point B should be replaced by point $\mathrm{H}$, the gas-oil cusp point as indicated in Figure 2. The appropriate region of the hodograph plane is shown in Figure 9(a). The two free interfaces, $\overline{H F}$ (corresponding to the gas-oil contact in petroleum engineering terminology), and $\overline{G F}$ (the water-oil contact), map to two semi-circles whose diameters depend on the relative densities of the gas, oil and water. Semi-circle $\overline{H F}$ has diameter $\mathrm{J}$ defined by

$$
J=\kappa\left(\gamma_{o}-\gamma_{g}\right) / \gamma_{o}
$$

while the diameter of semi-circle $\overline{G F}$ is the parameter $K$ defined in (10). The corresponding $\bar{\omega}$-plane is shown in Figure 9(b). The parameter $\Lambda$ shown in this figure is given by

$$
\Lambda=\frac{K}{J}=\frac{\gamma_{w}-\gamma_{o}}{\gamma_{o}-\gamma_{g}} .
$$

The auxiliary $\zeta$-plane used in the solution of this problem is shown in Figure 9 (c). Using the the Schwarz-Christoffel transformation, the $f$-plane is mapped onto the $\zeta$-plane via

$$
f(\zeta)=(Q /(2 \pi))[\log (\zeta+a)-\log (1-a)] .
$$

The $\bar{\omega}$-plane is mapped onto the $\zeta$-plane via

$$
\bar{\omega}(\zeta)=(2 / \pi)(1+\Lambda) \operatorname{arcsinh} \zeta^{1 / 2}-i \Lambda,
$$

and from this mapping the parameter $a$ in (43) is determined to be

$$
a=\sin ^{2}\left(\frac{\pi}{2} \frac{\Lambda}{\Lambda+1}\right) .
$$

Using (12), the expression relating the $z$-plane to the $\zeta$-plane is derived to be

$$
\frac{d z}{d \zeta}=-\frac{Q}{2 \pi K} \frac{1}{(\zeta+a)}\left[\frac{2}{\pi}(1+\Lambda) \operatorname{arcsinh} \zeta^{1 / 2}-i \Lambda\right] .
$$

The shape of the free boundaries $\overline{G F}$ and $\overline{H F}$ can be obtained by integrating (46) for $-\infty<\zeta<-1$ and $0<\zeta<\infty$, respectively. By putting $\zeta=-(1+\nu)$, 

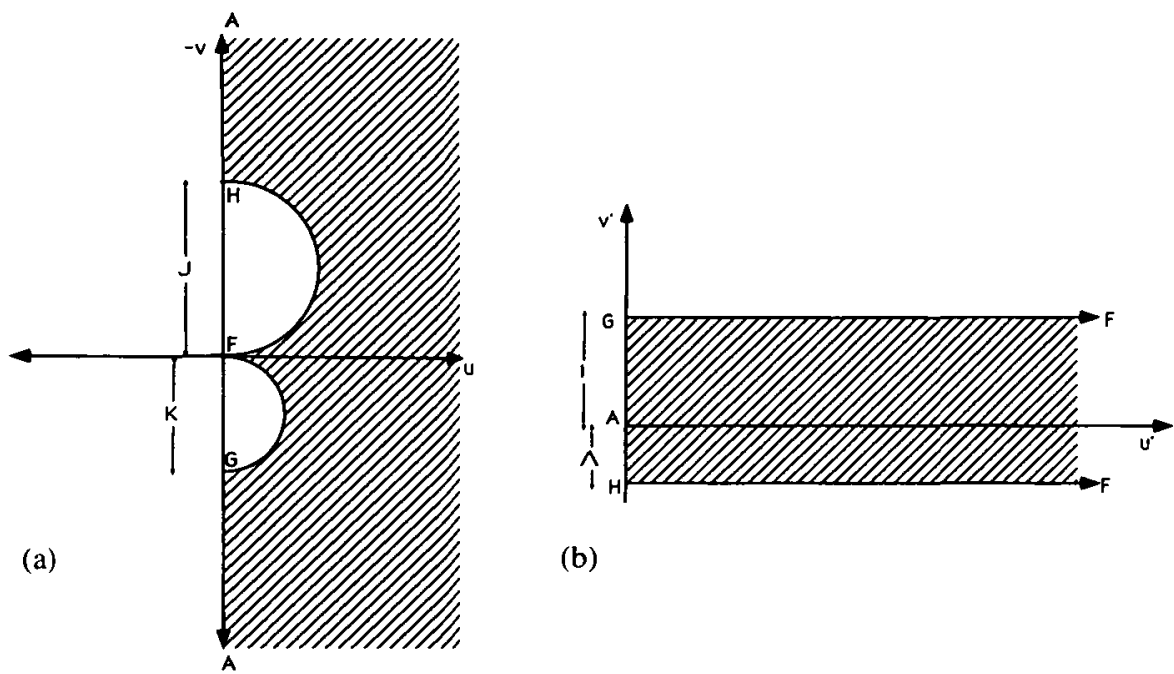

(b)

(c)

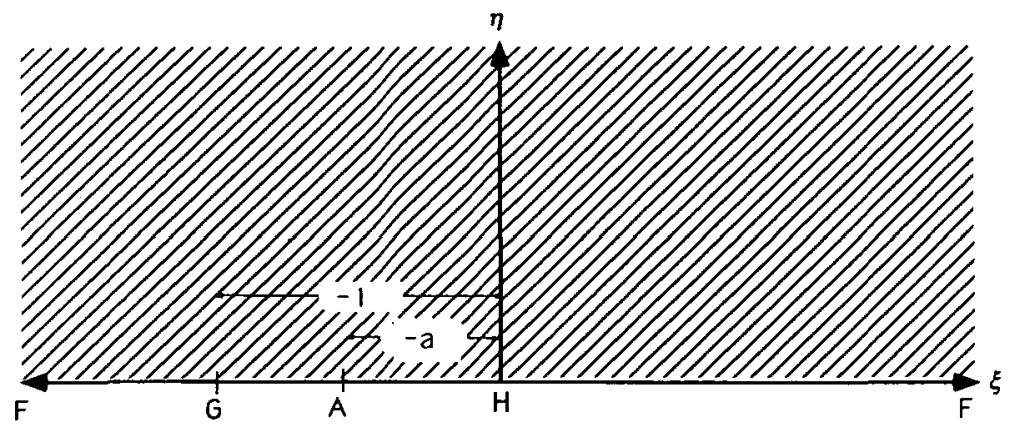

(d)

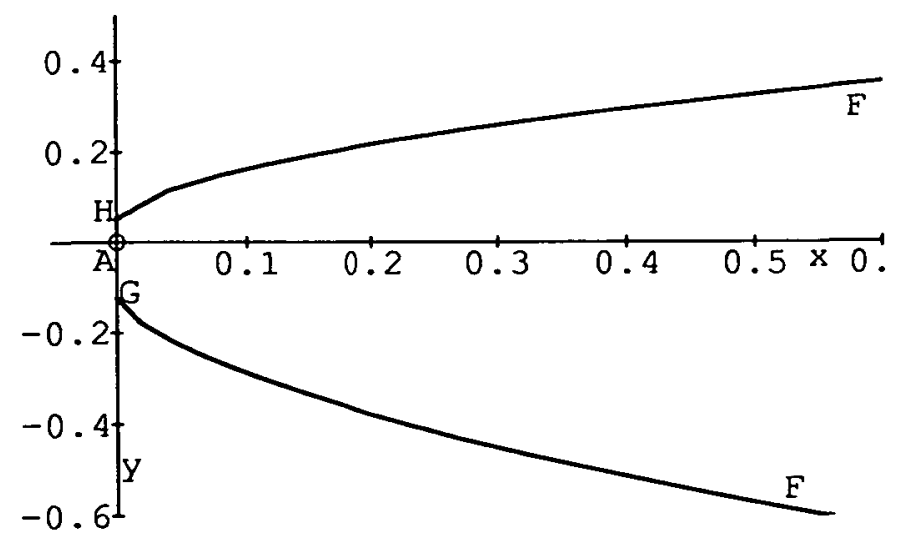

FIGURE 9. The problem of simultaneous gas and water cresting towards a horizontal well: (a) the $\omega$-plane, (b) the $\bar{\omega}$-plane, (c) the intermediate $\zeta$-plane, and (d) the calculated free interfaces in the z-plane for $a=0.25$. 
in one case, and $\zeta=\nu$, in the other, the parametric equations of the boundaries become

$$
\begin{aligned}
\frac{x_{G} K}{Q} & =\frac{(1+\Lambda)}{\pi^{2}} \int_{0}^{\nu} \frac{\operatorname{arcsinh} \nu^{1 / 2} d \nu}{\nu+(1-a)}, \\
\frac{y_{G} K}{Q} & =-\frac{1}{2 \pi} \log \frac{\nu+(1-a)}{(1-a)}
\end{aligned}
$$

and

$$
\begin{aligned}
& \frac{x_{H} K}{Q}=\frac{(1+\Lambda)}{\pi^{2}} \int_{0}^{\nu} \frac{\operatorname{arcsinh} \nu^{1 / 2} d \nu}{\nu+a}, \\
& \frac{y_{H} K}{Q}=\frac{\Lambda}{2 \pi} \log \frac{\nu+a}{a}
\end{aligned}
$$

where $0<\nu<\infty$ and $0 \leq a \leq 0.5$.

When $a=0, \Lambda=0$ and the problem reduces to that discussed in Section 2.2. When $a=0.5, \Lambda=1$ and the parametric equations of the free boundaries become degenerate. This corresponds to the case when $\gamma_{o}-\gamma_{g}=\gamma_{w}-\gamma_{o}$ and the crests are symmetrically distributed about a horizontal line through the sink at $A$.

\begin{tabular}{|c|c|c|c|c|}
\hline$a$ & $\Lambda$ & $\overline{A H}$ & $\overline{A G}$ & $\overline{A H}+\overline{A H}$ \\
\hline 0 & 0 & 0 & 0.2206 & 0.2206 \\
0.05 & 0.1676 & 0.0165 & 0.1530 & 0.1695 \\
0.10 & 0.2576 & 0.0256 & 0.1407 & 0.1663 \\
0.15 & 0.3390 & 0.0341 & 0.1331 & 0.1672 \\
0.20 & 0.4188 & 0.0426 & 0.1277 & 0.1702 \\
0.25 & 0.5000 & 0.0514 & 0.1234 & 0.1749 \\
0.30 & 0.5848 & 0.0609 & 0.1200 & 0.1808 \\
0.35 & 0.6751 & 0.0712 & 0.1170 & 0.1882 \\
0.40 & 0.7728 & 0.0826 & 0.1145 & 0.1971 \\
0.45 & 0.8801 & 0.0955 & 0.1123 & 0.2078 \\
0.50 & 1.0000 & 0.1103 & 0.1103 & 0.2206 \\
\hline
\end{tabular}

TABLE 4. Calculated values for the gas-oil cusp height, $\overline{A H}$, and the water-oil cusp height, $\overline{A G}$. 
The heights of the cusps above and below the sink, $\overline{A H}$ and $\overline{A G}$, can be found by integrating (46) for $-a<\zeta<0$ and $-1<\zeta<-a$, respectively. Table 4 shows the results obtained by performing these integrations numerically for different values of $a$. At $a=0, \overline{A H}=0$ and $\overline{A G}=0.2206$, as expected. At $a=0.5, \overline{A H}=\overline{A G}=0.1103$. The iriterfaces calculated for $a=0.25$ are shown in Figure 9(d).

The engineering problem of interest is to locate the optimum position of the sink with respect to the critical rate for cresting. Ideally, one would wish to be able to express the optimum height of the sink as a fraction of the height of the oil column. Unfortunately, because of the unphysical nature of the boundary conditions, the interfaces found by the hodograph method do not approach a horizontal surface (in fact, they follow the equation of a parabola at large distances from the sink [8]) so there is no clearly defined value for the height of the oil column. However, it is possible to find the limit of the ratio of the height of the sink below one interface to the total height between the two interfaces at large distances from the sink, i.e.

$$
\begin{aligned}
D & =\lim _{x \rightarrow \infty} \frac{\left(y_{H}+\overline{A H}\right)}{\left(y_{H}+\overline{A H}\right)+\left(y_{G}+\overline{A G}\right)} \\
& =\lim _{\nu \rightarrow \infty} \frac{\Lambda \log [(\nu+a) / a]}{\Lambda \log [(v+a) / a]+\log [(\nu+(1-a)) /(1-a)]} \\
& =\Lambda /(\Lambda+1) \\
& =\left(\gamma_{w}-\gamma_{o}\right) /\left(\gamma_{w}-\gamma_{g}\right) .
\end{aligned}
$$

By taking limits, this answer effectively neglects the cusp heights $\overline{A G}$ and $\overline{A H}$. Interestingly, it is the same answer as was derived by Meyer and Garder [10] for the optimum perforation position of a vertical well in a three-phase reservoir. In their analysis they assumed that the critical situation occurred when the gas and water cones just reached the perforations, so they too neglected the heights $\overline{A G}$ and $\overline{A H}$.

\section{Conclusion}

In this paper, a numerical technique has been used to find the shape of the free interface and values of critical heights for the case of water cresting towards a horizontal well beneath an impermeable plane. This extends previous work which was restricted to the cases of a sink in an unconfined oil zone and a sink 
on a horizontal plane, which can be solved analytically using the hodograph method for flow in porous media. A further extension has been made by applying the hodograph method to the problem of simultaneous gas and water cresting towards a horizontal well. Shapes of the gas-oil and water-oil interfaces have been found and an expression for the optimal placement of the well with respect to the interfaces given. This problem is of practical importance for oil production from thin oil column reservoirs.

\section{Acknowledgement}

I would like to thank Dr M. J. Landman for the use of his Newton solver program. I also thank The Broken Hill Proprietary Co. Ltd. for permission to publish this paper.

\section{References}

[1] J. Bear, Dynamics of fluids in porous media (American Elsevier, New York, 1972).

[2] J. Bear and G. Dagan, "Some exact solutions of interface problems by means of the hodograph method", J. Geophys. Res. 69 (1964) 1563-1572.

[3] J. R. Blake and A. Kucera, "Coning in oil reservoirs", Math. Scientist 13 (1988) 36-47.

[4] J. R. Blake, S. K. Lucas and A. Kucera, "A boundary-integral method applied to water coning in oil reservoirs", J. Aust. Math. Soc. Ser. B 32 (1991) 261-283.

[5] I. L. Collings, "Two infinite froude number cusped free surface flows due to a submerged line source or sink", J. Aust. Math. Soc. Ser. B 28 (1986) 260-270.

[6] J. Dupuit, Etudes théoriques et pratiques sur le mouvement des eaux, 2nd ed. (Dumont, Paris, 1863).

[7] S. Ekrann, "Production from thin oil zones", in Report no. SPT T-6/87, SPOR, Norway, 1987).

[8] F. M. Giger, "Analytic 2-D models of water cresting before breakthrough for horizontal wells", SPE Res. Eng. (November 1989) 409-416.

[9] G. C. Hocking, "Cusp-like free-surface flows due to a submerged source or sink in the presence of a flat or sloping bottom", J. Aust. Math. Soc. Ser. B 26 (1985) 470-486.

[10] H. I. Meyer and A. O. Garder, "Mechanics of two immiscible fluids in porous media", $J$. Appl. Phys. 25 (1954) 1400-1406.

[11] M. Muskat and R. B. Wyckoff, "An approximate theory of water coning in oil production", Trans. AIME 114 (1935) 144-163.

[12] P. Y. Polubarinova-Kochina, Theory of groundwater movement, English transl. by R. J. M. De Wiest, Princeton University Press, Princeton, N.J., 1962 (Gostek-hizdat, Moscow, 1952).

[13] E. O. Tuck and J. M. Vanden Broeck, "A cusp-like free-surface flow due to a submerged source or sink”, J. Aust. Math. Soc. Ser. B 25 (1984) 443-450.

[14] J. M. Vanden Broeck and J. B. Keller, "Free surface flow due to a sink", J. Fluid Mech. 175 (1987) 207-224. 